\title{
Physician Awareness of Knee and Hip Pain in the Context of Coronary Heart Disease Treatment
}

\author{
Mathias Glehr, ${ }^{1}$ Anna Kaltenbach, ${ }^{1}$ Reinhold Glehr, ${ }^{2}$ Patrick Sadoghi, ${ }^{1}$ \\ Andreas Leithner, ${ }^{1}$ Gerald Gruber, ${ }^{1}$ Joanna Szkandera, ${ }^{3}$ Sabine Ludt, ${ }^{4}$ Ines Vielgut, ${ }^{1}$ \\ Reingard Glehr, ${ }^{5}$ and Michel Wensing ${ }^{6}$
}

\author{
${ }^{1}$ Department of Orthopaedic Surgery, Medical University of Graz, Auenbruggerplatz 5, 8036 Graz, Austria \\ ${ }^{2}$ Medical University of Graz, Auenbruggerplatz 5, 8036 Graz, Austria \\ ${ }^{3}$ Division of Clinical Oncology, Department of Internal Medicine, Medical University of Graz, Auenbruggerplatz 5, 8036 Graz, Austria \\ ${ }^{4}$ Department of General Practice and Health Services Research, Voßstraße 2, Geb. 37, 69115 Heidelberg, Germany \\ ${ }^{5}$ Department of General Practice and Family Medicine, Medical University of Vienna, Kinderspitalgasse 15/1, 1090 Vienna, Austria \\ ${ }^{6}$ Scientific Institute for Quality of Healthcare, Radboud University Nijmegen Medical Centre, P.O. Box 9101, \\ 6500 HB Nijmegen, The Netherlands
}

Correspondence should be addressed to Gerald Gruber; gerald.gruber@klinikum-graz.at

Received 31 August 2013; Accepted 30 October 2013; Published 3 February 2014

Academic Editors: C. L. Athanasuleas, C. Carbucicchio, and F. Giallauria

Copyright (C) 2014 Mathias Glehr et al. This is an open access article distributed under the Creative Commons Attribution License, which permits unrestricted use, distribution, and reproduction in any medium, provided the original work is properly cited.

Background. The benefit of physical activity for the prevention and treatment of cardiovascular disease (CVD) has been well documented. The aim of the present study was to determine the level of awareness among general practitioners (GPs) of knee and hip problems in patients with CVD or CVD risk. Design. Cross-sectional questionnaire survey. Setting and Subjects. Thirty-five Austrian GPs and 1,118 patients were included. The GPs completed an extraction form about the presence or absence of documented evidence of problems related to the knee and/or hip joint within the patient medical data. Patients, in turn, were asked to complete a questionnaire that included the Oxford Knee/Hip Score and the cardiovascular risk-chart established by the European Society of Cardiology. Results. In 748 patients' data from medical records and questionnaires were available. $40.9 \%$ of these patients suffered from serious knee pain and 32.1\% from hip pain. However, in the medical records, in only $51.3 \%$ (knee) and $48.1 \%$ (hip) of these pain-patients the problems were documented. Conclusion. Joint disorders of the knee and hip problems are considerable barriers to effective physical activity and can therefore contribute to the development of CVD. Our data showed that GP awareness of such knee/hip disorders should be improved.

\section{Background and Objective}

The risk charts from the European Guidelines on Cardiovascular Disease Prevention focus on blood pressure, lipid profile, and smoking [1]. Regular physical activity and aerobic exercise training are recommended as a very important nonpharmacological treatment for primary and secondary CVD prevention [2-6]. However, to date, these guidelines have made no mention of the fact that osteoarthrotic pain in the knee and hip joint is a barrier to physical activity and should therefore be addressed during clinical consultation of CVD patients [1]. This is particularly problematic when one considers that patients with osteoarthritis seem to have higher CVD risk factors than the general population in all observed categories (hypertension, diabetes, high total cholesterol, low HDL cholesterol, and renal impairment or failure) [7].

Physical activity (PA) has been shown to inhibit the emergence and progression of CVD. It has beneficial effects on the development of atherosclerosis and results in a significant reduction in all-cause mortality [5]. Even in the more disabled patients, small amounts of properly supervised physical activity help improve cardiovascular status, maintain an independent lifestyle, and counteract disease-related 
TABLE 1: Demographic data of the patients.

\begin{tabular}{lccc}
\hline & CVD group & Risk group & Total \\
\hline$N$ total & 377 & 371 & 748 \\
Female & $129(34 \%)$ & $138(37 \%)$ & $267(36 \%)$ \\
Mean age (SD) & $69.43(10.88)$ & $64.71(10.20)$ & $67.09(10.54)$ \\
Mean BMI (SD) & $27.42(4.84)$ & $28.24(4.24)$ & $27.83(4.54)$ \\
Mean OKS (SD) & $34.13(9.66)$ & $36.56(8.21)$ & $35.33(8.94)$ \\
Mean OHS (SD) & $33.36(9.14)$ & $35.73(7.75)$ & $34.54(8.45)$ \\
\hline
\end{tabular}

Standard deviation (SD); body mass index (BMI).

depression $[8,9]$. However, most patients with CVD do not reach a sufficient level of PA because a necessary lifestyle change cannot be performed. Since many of these patients are elderly, pain in the knee or hip joint may present a barrier to the modification of behaviour or lifestyle [3]. Meta-analyses of clinical studies have confirmed the positive effect of PA in the rehabilitation and therapy for patients suffering from CVD, myocardial infarction, or stroke $[2,5]$, which led to recommendations on PA in the secondary prevention of CVD $[4,10]$.

The aim of the present study was to determine the level of awareness among GPs of knee and hip problems in patients with CVD or CVD risk.

\section{Methods}

Over 100 GPs were selected by the Austrian Society of General Medicine and asked if they want to take part on a scientific project. The study was part of an international research project on cardiovascular risk management in primary care [11-13]. The protocol for this prospective study was approved by the Ethics Committee of the Medical University of Graz, and a written informed consent was obtained from all participants included.

Two samples of patients were included: patients with a documented $\operatorname{CVD}(n=377$ patients $)$ and patients with a documented high risk of cardiovascular events $(n=371$ patients). The sample with established CVD included patients with documented myocardial infarction or angina pectoris, as well as patients who had already had a vascular surgery or intervention. The sample "patients at high risk of cardiovascular events" was defined by meeting 3 of the following 4 criteria in the GP's documentation records: hypertension, hypercholesterolemia, smoking, and men over 60 years of age. Exclusion criteria were diabetes mellitus, terminal illness, cognitive impairment, psychiatric illness, and language problems $[11,13]$.

The patients consulted the GPs for miscellaneous reasons. They were not invited by the GP. Anonymity was guaranteed by forwarding merely the identification number of the questionnaire and extraction form. Identification was enabled by assigning one identification number for each patient and one for each GP. The GPs completed an extraction form about the presence or absence of documented evidence of problems related to the knee and/or hip joint within the patient medical data. Each patient had at least one consultation at the GP in the week before the GP completed the extraction form.

The patient's questionnaire was filled up by the patient at the day of visiting the GP. It contained items about joint status, restricted to the knee and hip. Symptoms in these joints were measured by the Oxford Scores, which are available for both knee-the Oxford Knee Score (OKS) - and hip-the Oxford Hip Score (OHS) [14-16]. Oxford Scores range from 0 to 48, with 0 indicating the worst functional joint status and 48 indicating the best. A total OKS or OHS between 30 and 39 points indicates mild or moderate knee or hip arthritis, while scores under 30 points represent severe osteoarthritis (OA) [14-16]. When assessing the prevalence of joint disorders in the three different groups, knee and hip disorders were evaluated separately. Patients were asked if they had had knee or hip pain in the past four weeks. Those who approved this condition were asked to complete the 12 questions of the Oxford Knee/Hip Score.

2.1. Statistical Methods. Documentation of the GP (patient suffering from knee or hip pain) was correlated with the $\mathrm{OKS}$ and OHS in the patient's questionnaire. The median value was used to evaluate the OKS and OHS scores because the distribution was not normal and the scores were based on a questionnaire (ordinal scale) for which the median is a more representative measure. As a measure of variation, the interquartile range (IQR) was calculated. For further statistical analysis, either Spearman's rank correlations or the Kruskal-Wallis tests were performed. Linear regression analyses were used to identify relationships between one dependent variable and one or more independent (predictor) variables. Linear regressions were used due to the fact that this analysis is a robust statistic which is resistant to deviations from assumptions. A $P$ value $<0.05$ was considered to be statistically significant. Statistical analysis was performed with SPSS software version 19 (SPSS, Chicago, IL; 2011).

\section{Results}

Thirty-eight Austrian GPs participated in the study-data from 35 GPs could be used because a lack of data quality from 3 GPs. Those 35 GPs provided data from 748 patients (Table 1). Some of the patients did not fulfil the complete questionnaire; for example, they did not write down their age. 
TABLE 2: Documentation of knee and hip pain by the GP in patients with documented CVD or documented risk for cardiovascular events.

\begin{tabular}{|c|c|c|c|c|}
\hline \multirow{2}{*}{ Group } & \multicolumn{2}{|c|}{ Knee pain } & \multicolumn{2}{|c|}{ Hip pain } \\
\hline & Yes & No & Yes & No \\
\hline CVD documented & 167 & 210 & 126 & 251 \\
\hline CVD risk group & 139 & 232 & 114 & 257 \\
\hline$\sum$ & 306 & 442 & 240 & 508 \\
\hline Pain documented by the GP & $157(\mathbf{5 1 . 3 \%})$ & & $3(47.1$ & \\
\hline
\end{tabular}

These missing files are mentioned in Table 1, demographic data.

3.1. Documentation of Knee and Hip Pain. In the study, 306 patients $(40.9 \%$ of the CVD and CVD risk group) indicated (in our dichotomous item) that they had had knee pain during the last four weeks (Table 2). In 157 of these patients $(51.3 \%)$, the GP had documented in the medical record that these patients suffer from knee pain. For the hip joint, 240 patients (32.1\% of the CVD and CVD risk group) indicated that they had pain during the last four weeks. In the GPs' medical files, hip pain was documented in 113 patients (47.1\%). There were patients with both knee and hip pain. The data of these patients were used for both knee and hip analysis because the Oxford Knee and Hip Scores are specially designed for one joint and ask for specific impairment that comes along with this joint.

No associations were found between the documentation of joint-related problems and the GP's professional experience, gender, or location (rural or urban area with 100,000 inhabitants or more).

The OKS was 36 in CVD group, $(\mathrm{IQR}=14)$ and 37 in risk group $(\mathrm{IQR}=11)$. The median OHS was statistically significantly different in the two groups (CVD group 35 $(\mathrm{IQR}=13)$ and risk group $37(\mathrm{IQR}=10))$. Women had a significantly lower OKS $(P=0.02)$. There was no gender difference regarding the OHS.

\section{Discussion}

The aim of this study was to determine the level of awareness among general practitioners of knee and hip problems in patients with CVD or CVD risk. The GP is the primary contact for arthritis patients and the main care provider for most patients [17]. In our study, the participating GPs were only aware of patients' pain related to knee or hip problems in roughly half of the cases.

Prior studies also deal with joint-related pain in the lower extremity as a risk for CVD or GP's awareness of pain [1822]. Regarding awareness of pain in a publication of Peters et al. $45 \%$ of the patients reported about knee problems, $24 \%$ about hip problems, and $31 \%$ about both [23]. In a large population based cohort study of Nüesch et al. $28 \%$ of the responders reported about pain in the knee and hip joint. They concluded that people with osteoarthritis of the knee and hip have an excess all-cause mortality compared with the general population, particularly pronounced for death from cardiovascular causes [24]. Marzolini et al. reported that over half $(56 \%)$ of the patients with coronary artery diseases had musculoskeletal problems with joint pain accounting for $64.4 \%$ of these. Despite a greater need for comprehensive risk factor management in patients with musculoskeletal conditions, fewer patients were referred to cardiac rehabilitation [25]. Philbin et al. found that patients with lower extremity osteoarthritis may be at advanced risk for the development of cardiovascular heart disease by virtue of their unfavourable risk factor profile [26].

The "European Guidelines on Cardiovascular Disease prevention in clinical practice" mention both regular physical activity and aerobic exercise training to reduce the risk of fatal and nonfatal coronary events in healthy individuals, subjects with coronary risk factors, and cardiac patients over a wide age range [1]. However, these guidelines fail to mention that, in some cases, the patient may be unable to perform regular physical activity and exercise training because of pain in the lower extremities. When caring for patients with chronic diseases, doctors should remain alert to other disorders and minimize the number of missed opportunities for treating them [27]. This should also be highlighted in the guidelines for CVD prevention.

\section{Limitations}

This study has several limitations. It might be sometimes common for older people to normalise joint pain and not report to the GP [28]. This lack of information could not be evaluated in this study. Also the potential ceiling effect of the OKS and OHS may have affected the current study, since patients can have musculoskeletal limitations without OKS or OHS identifying these.

\section{Conclusion}

Joint disorders of the knee and hip are considerable barriers to effective physical activity and can be considered as an independent CVD risk factor. Yet our data showed that there is considerable room for improvement in the GP awareness of such joint disorders when treating CVD. The guidelines on CVD prevention should therefore mention pain in the knee and hip joint as a barrier to physical activity and the related need to address joint problems during treatment for CVD. 
Further studies are necessary to evaluate joint disorders as CVD risk factor.

\section{Ethical Approval}

The protocol for this prospective study was approved by the Ethics Committee of the Medical University of Graz (registration no. 19-161 ex 07/08).

\section{Conflict of Interests}

None of the authors received any financial support or other benefits from commercial sources for the work reported on in the submitted paper. None of the authors have any financial interests that could create a potential conflict of interest, or the appearance of a conflict of interests with regard to the submitted work.

\section{Acknowledgments}

The authors are grateful to the Austrian Society of General Practice and Family Medicine for supporting the study. Special thanks are due to Hans-Christian Caluba for data acquisition. Bertelsmann Foundation (Gütersloh, Germany) initiated, coordinated, and funded the EPA Cardio project [11] in collaboration with IQ Healthcare of the Radboud University Nijmegen (The Netherlands).

\section{References}

[1] J. Perk, G. de Backer, H. Gohlke et al., "European guidelines on cardiovascular disease prevention in clinical practice (version 2012). The Fifth Joint Task Force of the European Society of Cardiology and Other Societies on Cardiovascular Disease Prevention in Clinical Practice (constituted by representatives of nine societies and by invited experts)," European Heart Journal, vol. 33, no. 13, pp. 1635-1701, 2012.

[2] D. A. Brown, K. N. Jew, G. C. Sparagna, T. I. Musch, and R. L. Moore, "Exercise training preserves coronary flow and reduces infarct size after ischemia-reperfusion in rat heart," Journal of Applied Physiology, vol. 95, no. 6, pp. 2510-2518, 2003.

[3] M. Hendry, N. H. Williams, D. Markland, C. Wilkinson, and P. Maddison, "Why should we exercise when our knees hurt? A qualitative study of primary care patients with osteoarthritis of the knee," Family Practice, vol. 23, no. 5, pp. 558-567, 2006.

[4] J. Li and J. Siegrist, "Physical activity and risk of cardiovascular disease-a meta-analysis of prospective cohort studies," International Journal of Environmental Research and Public Health, vol. 9, no. 2, pp. 391-407, 2012.

[5] R. S. Taylor, A. Brown, S. Ebrahim et al., "Exercise-based rehabilitation for patients with coronary heart disease: systematic review and meta-analysis of randomized controlled trials," The American Journal of Medicine, vol. 116, no. 10, pp. 682-692, 2004.

[6] K. K. Danielsen, M. Svendsen, S. Maehlum, and J. SundgotBorgen, "Changes in body composition, cardiovascular disease risk factors, and eating behavior after an intensive lifestyle intervention with high volume of physical activity in severely obese subjects: a prospective clinical controlled trial," Journal of Obesity, vol. 2013, Article ID 325464, 12 pages, 2013.
[7] G. Singh, J. D. Miller, F. H. Lee, D. Pettitt, and M. W. Russell, "Prevalence of cardiovascular disease risk factors among US adults with self-reported osteoarthritis: data from the third National Health and Nutrition Examination Survey," The American Journal of Managed Care, vol. 8, no. 15, pp. S383-S391, 2002.

[8] S. M. McCurry, M. Von Korff, M. V. Vitiello et al., "Frequency of comorbid insomnia, pain, and depression in older adults with osteoarthritis: predictors of enrollment in a randomized treatment trial," Journal of Psychosomatic Research, vol. 71, no. 5, pp. 296-299, 2011.

[9] D. L. Riddle, X. Kong, and G. K. Fitzgerald, "Psychological health impact on 2-year changes in pain and function in persons with knee pain: data from the osteoarthritis initiative," Osteoarthritis and Cartilage, vol. 19, no. 9, pp. 1095-1101, 2011.

[10] T. K. Jorgensen, M. Nordentoft, and J. Krogh, "How do general practitioners in Denmark promote physical activity?" Scandinavian Journal of Primary Health Care, vol. 30, no. 3, pp. 141-146, 2012.

[11] M. Wensing, S. Ludt, S. Campbell, J. van Lieshout, E. Volbracht, and R. Grol, "European practice assessment of cardiovascular risk management (EPA cardio): protocol of an international observational study in primary care," Implementation Science, vol. 4, article 3, 2009.

[12] S. Ludt, S. M. Campbell, J. van Lieshout, R. Grol, J. Szecsenyi, and M. Wensing, "Development and pilot of an internationally standardized measure of cardiovascular risk management in European primary care," BMC Health Services Research, vol. 11, article 70, 2011.

[13] J. van Lieshout, R. Grol, S. Campbell et al., "Cardiovascular risk management in patients with coronary heart disease in primary care: variation across countries and practices: an observational study based on quality indicators," BMC Family Practice, vol. 13, article 96, 2012.

[14] J. Dawson, R. Fitzpatrick, A. Carr, and D. Murray, "Questionnaire on the perceptions of patients about total hip replacement," Journal of Bone and Joint Surgery B, vol. 78, no. 2, pp. 185-190, 1996.

[15] J. Dawson, R. Fitzpatrick, D. Murray, and A. Carr, "Questionnaire on the perceptions of patients about total knee replacement," Journal of Bone and Joint Surgery B, vol. 80, no. 1, pp. 63-69, 1998.

[16] D. W. Murray, R. Fitzpatrick, K. Rogers et al., "The use of the Oxford hip and knee scores," Journal of Bone and Joint Surgery B, vol. 89, no. 8, pp. 1010-1014, 2007.

[17] T. Rosemann, T. Körner, M. Wensing et al., "Rationale, design and conduct of a comprehensive evaluation of a primary care based intervention to improve the quality of life of osteoarthritis patients. The PraxArt-project: a cluster randomized controlled trial [ISRCTN87252339]," BMC Public Health, vol. 5, article 77, 2005.

[18] J. M. Cramm and A. P. Nieboer, "Factorial validation of the patient assessment of chronic illness care (PACIC) and PACIC short version (PACIC-S) among cardiovascular disease patients in the Netherlands," Health and Quality of Life Outcomes, vol. 10, article 104, 2012.

[19] C. Sanders, J. L. Donovan, and P. A. Dieppe, "Unmet need for joint replacement: a qualitative investigation of barriers to treatment among individuals with severe pain and disability of the hip and knee," Rheumatology, vol. 43, no. 3, pp. 353-357, 2004 . 
[20] G. C. Davis, M. L. Hiemenz, and T. L. White, "Barriers to managing chronic pain of older adults with arthritis," Journal of Nursing Scholarship, vol. 34, no. 2, pp. 121-126, 2002.

[21] M. A. M. Gignac, A. M. Davis, G. Hawker et al., “"What do you expect? You're just getting older": a comparison of perceived osteoarthritis-related and aging-related health experiences in middle- and older-age adults," Arthritis Care and Research, vol. 55, no. 6, pp. 905-912, 2006.

[22] D. C. Ang, K. Thomas, and K. Kroenke, "An exploratory study of primary care physician decision making regarding total joint arthroplasty," Journal of General Internal Medicine, vol. 22, no. 1, pp. 74-79, 2007.

[23] T. J. Peters, C. Sanders, P. Dieppe, and J. Donovan, "Factors associated with change in pain and disability over time: a community-based prospective observational study of hip and knee osteoarthritis," British Journal of General Practice, vol. 55, no. 512, pp. 205-211, 2005.

[24] E. Nüesch, P. Dieppe, S. Reichenbach, S. Williams, S. Iff, and P. Jüni, "All cause and disease specific mortality in patients with knee or hip osteoarthritis: population based cohort study," British Medical Journal, vol. 342, article d1165, 2011.

[25] S. Marzolini, P. I. Oh, D. Alter, D. E. Stewart, and S. L. Grace, "Musculoskeletal comorbidities in cardiac patients: prevalence, predictors, and health services utilization," Archives of Physical Medicine and Rehabilitation, vol. 93, no. 5, pp. 856-862, 2012.

[26] E. F. Philbin, M. D. Ries, G. D. Groff, K. A. Sheesley, T. S. French, and T. A. Pearson, "Osteoarthritis as a determinant of an adverse coronary heart disease risk profile," Journal of Cardiovascular Risk, vol. 3, no. 6, pp. 529-533, 1996.

[27] D. A. Redelmeier, S. H. Tan, and G. L. Booth, "The treatment of unrelated disorders in patients with chronic medical diseases," The New England Journal of Medicine, vol. 338, no. 21, pp. 15161520, 1998.

[28] J. Grime, J. C. Richardson, and B. N. Ong, "Perceptions of joint pain and feeling well in older people who reported being healthy: a qualitative study," British Journal of General Practice, vol. 60 , no. 577 , pp. 597-603, 2010. 


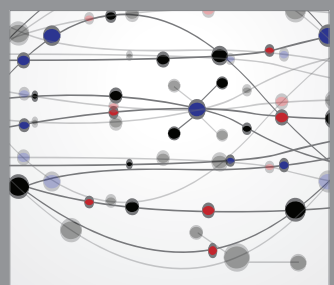

The Scientific World Journal
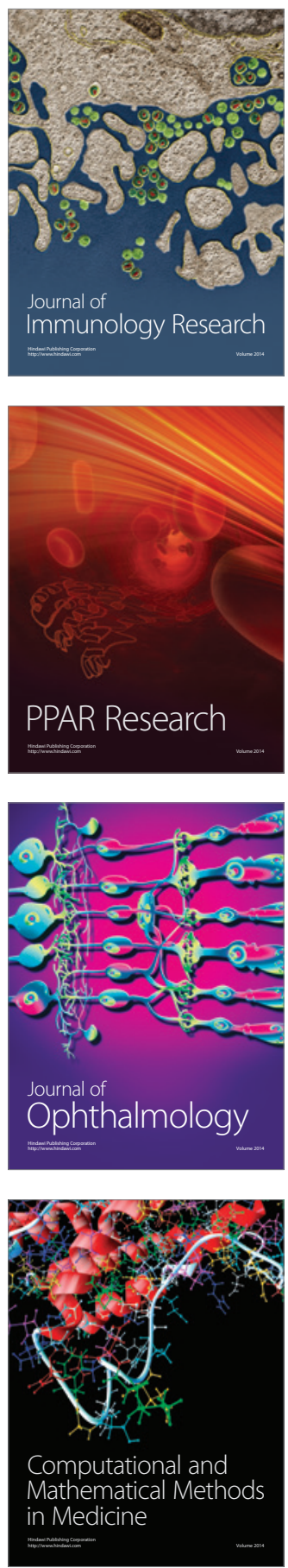

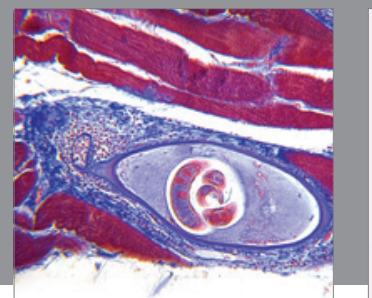

Gastroenterology

Research and Practice
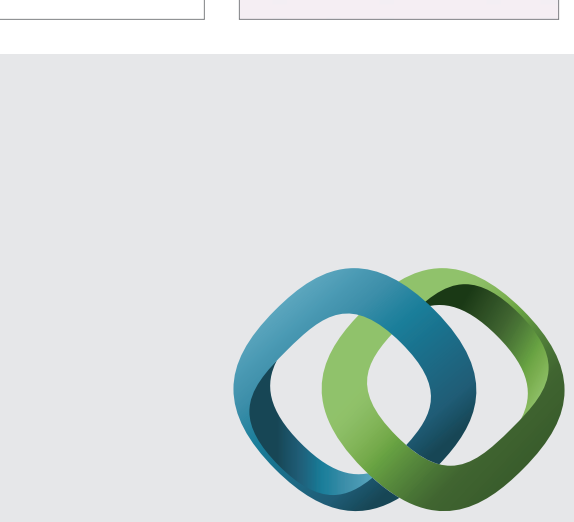

\section{Hindawi}

Submit your manuscripts at

http://www.hindawi.com
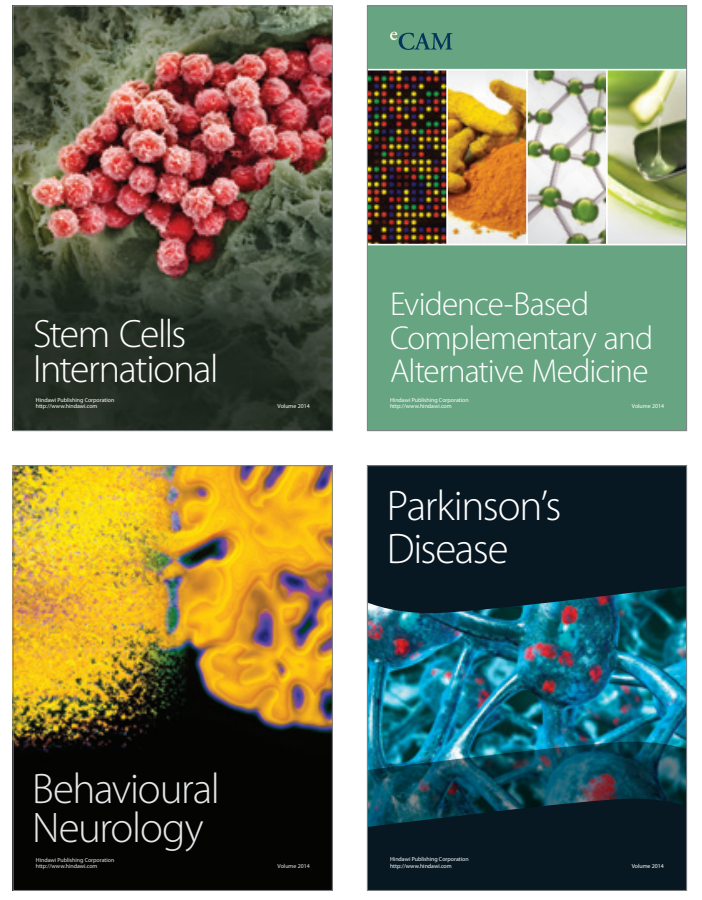
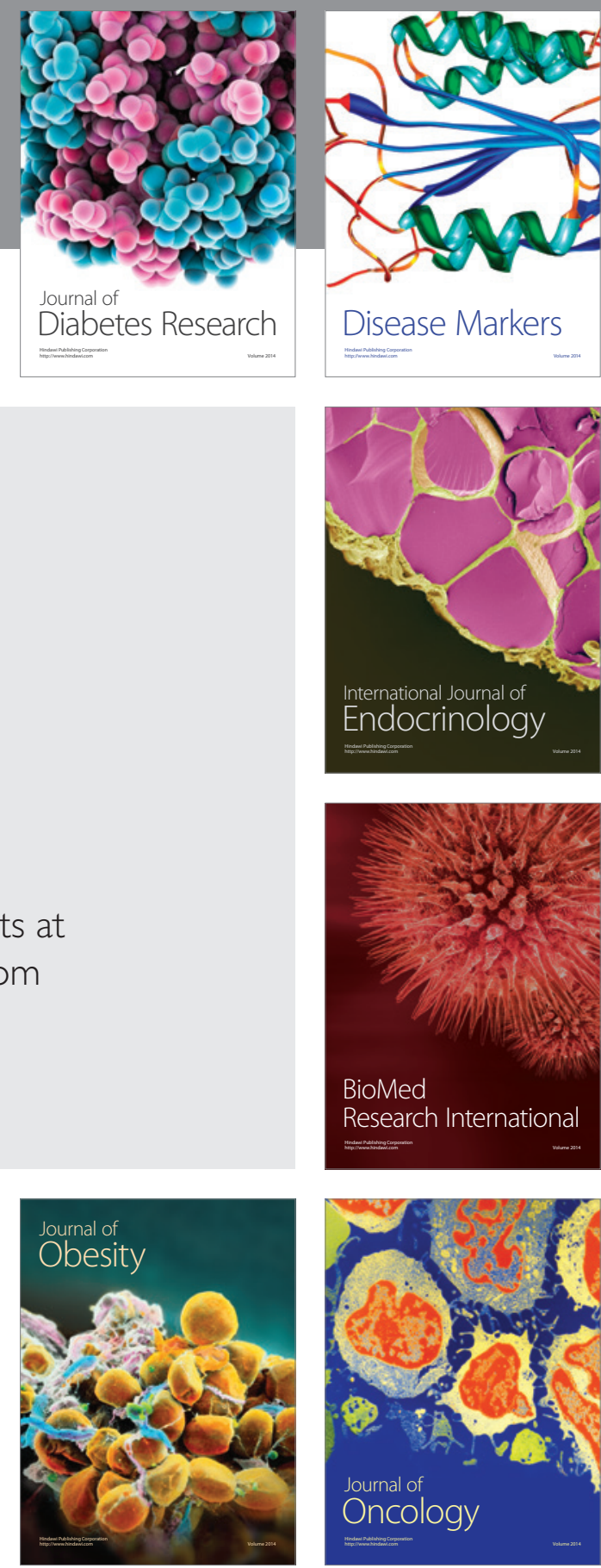

Disease Markers
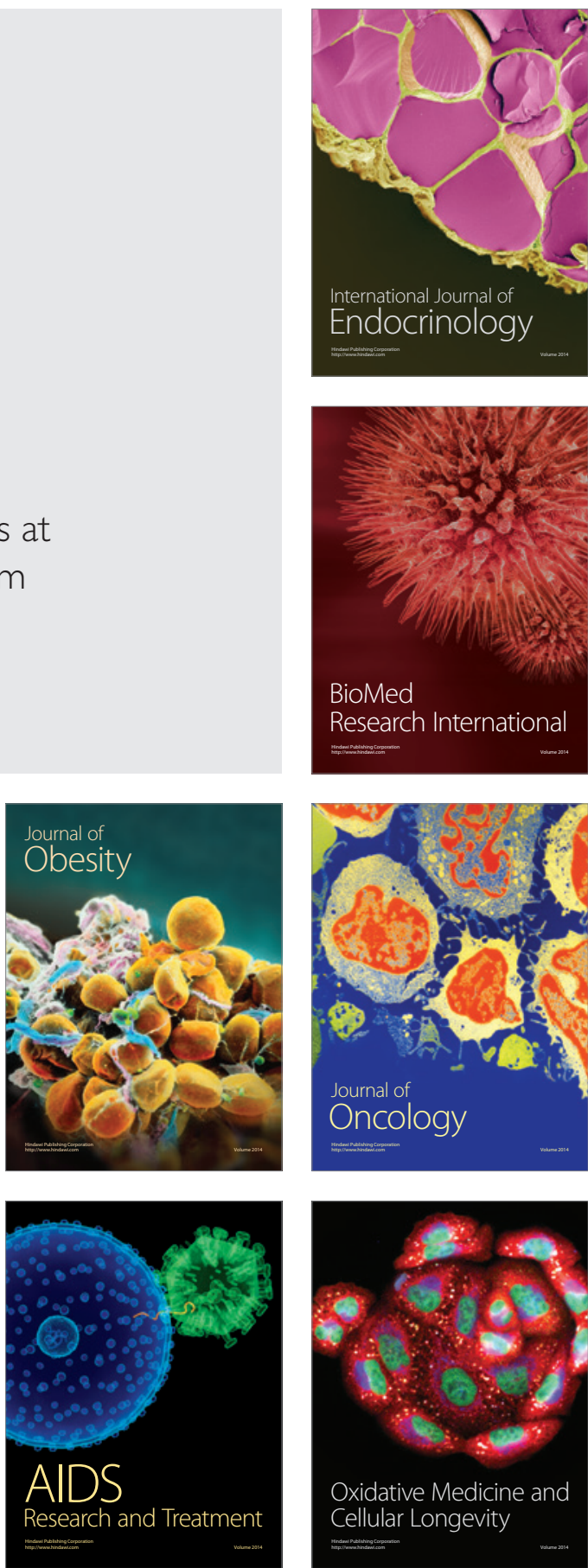